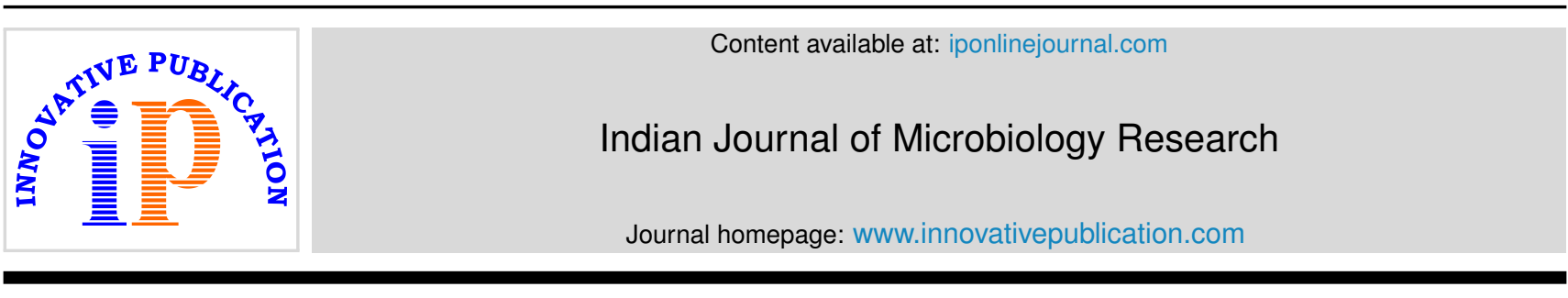

Original Research Article

\title{
Septicemia in neonates admitted to NICU with special reference to Acinetobacter species
}

\author{
Ruturaj M Kolhapure ${ }^{1}$, A. Ravi Shankar Reddy ${ }^{1, *}$, Raj Kumar H.R.V ${ }^{1}$ \\ ${ }^{1}$ Dept. of Microbiology, Kamineni Academy of Medical Sciences and Research Centre, Hyderabad, Telangana, India
}

\section{A R T I C L E I N F O}

\section{Article history:}

Received 28-04-2019

Accepted 24-06-2019

Available online 09-09-2019

Keywords:

Acinetobacter

Neonates

Septicemia

\begin{abstract}
A B S T R A C T
Introduction: Acinetobacter species are opportunistic pathogen, which are gaining importance in hospital acquired neonatal septicemia due to multidrug resistance and causing increased morbidity as well as mortality.

Materials and Methods: A prospective analysis was performed over a period of 6 months. Blood samples from neonates suspected of sepsis were collected and culture was done using conventional techniques. Acinetobacter when isolated were identified up to species level and drug sensitivity pattern of those isolate were done. Risk factors leading to Acinetobacter septicemia were also studied.

Results: Out of 200 blood culture samples, 12(17.1\%) Acinetobacter species were isolated, among these the predominant isolate was 7(58.3\%) Acinetobacter baumannii, followed by Acinetobacter calcoaceticus $3(25 \%)$ and $1(8.3 \%)$ each were A.lwofii and A.hemolyticus. All isolates were resistant to 3 or more group of drugs.

Conclusion: Neonatal septicemia especially with MDR Acinetobacter is on the rise and is associated with increased morbidity and mortality. Continuous surveillance of isolates from neonatal septicemia, adherence to infection control policies and rational antibiotic usage will reduce the incidence of such infections.
\end{abstract}

(C) 2019 Published by Innovative Publication.

\section{Introduction}

The genus Acinetobacter, now a member of family Moraxellaceae, consists of 25 DNA homology groups or genomospecies. Only 11 have been officially named; the two species most commonly seen in clinical specimens are Ainetobacter baumannii complex, the glucose-oxidizing non-hemolytic strain, and A.lwoffii, the glucose-negative, non-hemolytic strain. Most hemolytic strains are A.hemolyticus. Acinetobacter are ubiquitous in environment in soil, water, and foodstuffs. In the hospital environment, they have been associated with ventilators, humidifiers, catheters, and other devices.

About $25 \%$ of adults carry the organism on their skin, and about $7 \%$ carry the organism in their pharynx. If not already harboring Acinetobacter spp., hospitalized patients become easily colonized. In the past, when Acinetobacter spp. were isolated from nonsterile sites

\footnotetext{
* Corresponding author.

E-mail address: sourcewop@gmail.com (A. R. S. Reddy).
}

such as urine and many different types of respiratory specimens, they were usually considered insignificant colonizers or contaminants. However, with increased isolates of Acinetobacter that demonstrate resistance to most antimicrobial agents, including the carbapenems, their clinical significance when isolated from blood culture, cannot be dismissed as contaminants.

Acinetobacter spp. are opportunists, accounting for 3$5 \%$ of all hospital acquired infections; they are second only to P.aeruginosa in frequency of isolation of all nonfermenters in clinical microbiology lab. ${ }^{1}$

All Acinetobacter spp. are strictly aerobic, and they appear as gram-negative coccobacilli or even gram-negative cocci on Gram stain. Acinetobacter species can also appear as gram-positive cocci in smear made from blood culture bottles. ${ }^{1}$

Septicemia in neonates is a significant cause of morbidity and mortality in developing countries. Common isolates are Klebsiella spp. Staphylococcus aureus, Pseudomonas spp., and Enterobacter spp., Acinetobacter species are important 
potential pathogens in neonatal septicemia because of frequent colonization of ICUs and multi-drug resistance. ${ }^{2,3}$

A number of studies have reported the risk factor of infections from resistant strains of Acinetobacter species like prior antibiotic use, longer duration of intensive care unit stay, preterm birth, birth weight $<1500 \mathrm{~g}$. 3,4

The present study shows importance of Acinetobacter spp. as pathogen in neonatal blood stream infection. Identification of risk factors for Acinetobacter septicemia and antibiotic susceptibility testing were other objectives.

\section{Materials and Methods}

This prospective study was conducted in the department of Microbiology at Kamineni Institute of Medical Sciences, Narketpally, Telangana, over a period of 6 months from April 2013 to September 2013. The present study included 200 blood culture samples from suspected neonatal septicemia cases admitted to NICU. Samples were collected with aseptic precautions. These were processed using conventional bacteriological procedure for isolation of Acinetobacter species. Blood specimens were cultured using manual blood culture bottles containing $20 \mathrm{ml}$ of brain heart infusion broth with sps (sodium polyanetholsulphonate $0.025 \%$ ). $1 \mathrm{ml}$ of blood was inoculated with all due precautions. Blood cultures were considered negative only after 7 days of incubation.

Gram stain was carried out after 24 hours of incubation, followed by inoculation onto blood agar and MacConkey agar, and incubated aerobically for 24 hours at $37^{0} \mathrm{C}$.

Acinetobacter species identification was made with the help of phenotypic criteria by Gerner-Smidt, they included parameters like Gram stain, colony morphology, penicillin susceptibility, oxidase, catalase and urease activity, citrate reduction, glucose and lactose oxidative utilization, chloramphenicol sensitivity, and growth at $37^{0} \mathrm{C}$ and $44^{0} \mathrm{C}^{5}$ Antibiotic susceptibility testing was done by conventional disc diffusion method according to CLSI guidelines. ${ }^{6}$ Antibiotic discs used were Piperacillin $(100 \mu \mathrm{g})$, Ampicillin- sulbactam $(10 / 10 \mu \mathrm{g})$, Ceftazidime $(30 \mu \mathrm{g})$, Piperacillin-tazobactam Imipenem $(10 \mu \mathrm{g}), \quad$ Meropenem $(10 \mu \mathrm{g}), \quad \mathrm{G}$ entamicin $(10 \mu \mathrm{g})$, Amikacin $(30 \mu \mathrm{g})$, Netilmicin $(30 \mu \mathrm{g}), \quad \mathrm{C}$ iprofloxacin $(5 \mu \mathrm{g})$, Colistin $(10 \mu \mathrm{g})$ and Co-trimoxazole $(1.25 / 23.75$ $\mu \mathrm{g})$. ATCC 25922 Escherichia coli was used for quality control of anti biotic susceptibility testing. ${ }^{6}$

In the present study MDR Acinetobacter species would be defined as resistant to at least three groups of antibiotic agents such as penicillins and cephalosporins (including beta lactam inhibitor combination), fluroquinolones, and aminoglycosides.

\section{Results}

Out of 200 blood culture samples included in the study, $70(35 \%)$ were positive for aerobic bacteriological culture. Out of which 12(17.1\%) were due to Acinetobacter species. Among these $7(58.3 \%)$ were Acinetobacter baumanii, $3(25 \%)$ were Acinetobacter calcoaceticus and $1(8.3 \%)$ each were A.lwofii and A.hemolyticus. Table 1 .

The main risk factors associated with Acinetobacter neonatal septicemia were, hospital births $(100 \%)$, birth weight $<1500 \mathrm{~g}(75 \%)$, preterm birth $(58.3 \%)$, prolonged intravenous antibiotic use $(83.3 \%)$ and prolonged hospital stay $(66.6 \%)$. Table 2

All the 12(17.1\%) isolates were resistant to 3 or more group of drugs (Multi-drug resistant strain). The resistant percentage among various drugs were Piperacillin (100\%), Ampicillin- sulbactam (58.3\%), Ceftazidime (100\%), Piperacillin-tazobactam $(100 / 10 \mu \mathrm{g})(91.6 \%)$, Imipenem (58.3\%), Meropenem (58.3\%), Gentamicin (100\%), Amikacin (100\%), Netilmicin (66.6\%), Ciprofloxacin $(100 \%)$ and Co- trimoxazole $(100 \%)$, Colistin $(0 \%)$. Table 3 .

A high degree of resistant pattern was seen to various groups of antibiotics. All Acinetobacter baumannii complex (A.baumannii + A.calcoaciticus) isolate showed resistant to multiple group of antibiotics. All Acinetobacter baumannii isolates were resistant to Carbapenems (58.3\%). In comparison, both A.lwofii and A.hemolyticus were more susceptible to antibiotics used in the study. Many isolates showed increased sensitivity to Ampicillin- sulbactam, probably due to intrinsic sensitivity of Acinetobacter species to sulbactam. In our study we also saw increase in netilmicin sensitivity (33.4\%), among carbapenem resistant Acinetobacter baumannii isolates, for reasons unknown.

Table 1: Species of Acinetobacter isolated $(\mathrm{n}=12)$

\begin{tabular}{lll}
\hline Acinetobacter species & No. of isolates & Percentage \\
Acinetobacter baumannii & 7 & $58.3 \%$ \\
Acinetobacter calcoaceticus & 3 & $25 \%$ \\
Acinetobacter lwoffi & 1 & $8.3 \%$ \\
Acinetobacter haemolyticus & 1 & $8.3 \%$ \\
Total & 12 & $100 \%$ \\
\hline
\end{tabular}

Table 2: Risk factors for Neonatal sepsis $(n=12)$

\begin{tabular}{ll}
\hline Risk Factors & No. of Neonates $(\%)$ \\
Hospital birth & $12(100 \%)$ \\
Preterm birth & $7(58.3 \%)$ \\
Birth weight $<1500 \mathrm{~g}$ & $9(75 \%)$ \\
Prolonged hospital stay & $8(66.6 \%)$ \\
Prolonged intravenous antibiotic use & $10(83.3 \%)$ \\
\hline
\end{tabular}


Table 3: Resistant pattern of Acinetobacter species against various antimicrobial agents

\begin{tabular}{|c|c|c|c|c|}
\hline Antibiotics & A.baumannii $(\mathbf{n}=7)$ & A.calcoaceticus $(\mathbf{n}=\mathbf{3})$ & A.lwoffii $(\mathbf{n}=\mathbf{1})$ & A.hemolyticus $(\mathbf{n}=\mathbf{1})$ \\
\hline Piperacillin & $7(100 \%)$ & $3(100 \%)$ & $1(100 \%)$ & $1(100 \%)$ \\
\hline Gentamicin & $7(100 \%)$ & $3(100 \%)$ & $1(100 \%$ & $1(100 \%)$ \\
\hline Amikacin & $7(100 \%)$ & $3(100 \%)$ & $1(100 \%$ & $1(100 \%)$ \\
\hline Netilmicin & $3(42.85 \%)$ & $2(66.6 \%)$ & 0 & 0 \\
\hline Ceftazidime & $7(100 \%)$ & $3(100 \%)$ & $1(100 \%$ & $1(100 \%)$ \\
\hline Ciprofloxacin & $7(100 \%)$ & $3(100 \%)$ & $1(100 \%$ & $1(100 \%)$ \\
\hline Ampicillin-Sulbactam & $3(42.85 \%)$ & $2(66.6 \%)$ & 0 & 0 \\
\hline Piperacillin-Tazobactam & $7(100 \%)$ & $3(100 \%)$ & 0 & $1(100 \%)$ \\
\hline Imipenem & $5(71.4 \%)$ & $2(66.6 \%)$ & 0 & 0 \\
\hline Meropenem & $5(71.4 \%)$ & $2(66.6 \%)$ & 0 & 0 \\
\hline Co-trimoxazole & $7(100 \%)$ & $3(100 \%)$ & $1(100 \%$ & $1(100 \%)$ \\
\hline Colistin & 0 & 0 & 0 & 0 \\
\hline
\end{tabular}

\section{Discussion}

Acinetobacter species has evolved as an important opportunistic pathogen in healthcare settings, globally. It has remarkable ability to acquire multiple antibiotic resistance and to survive for prolonged periods under various environmental conditions, due to which it causes frequent hospital outbreaks. The common targets are critically ill patients with breach in skin integrity causing pneumonia, urinary tract infection, wound infection and septicemia. ${ }^{1-3,7}$

MDR Acinetobacter sp. septicemia in neonates is associated with high mortality. The present study was undertaken to find incidence and antibiotic resistance pattern of Acinetobacter sp. in neonatal septicemia.

In our study Acinetobacter neonatal septicemia incidence was $17.1 \%$. This was similar to the studies conducted by Asifa Nazir (13.7\%), Vinodkumar et al (9\%), Arora (12.3\%), and Mondal et al. (15.2\%). ${ }^{8-11}$ A.baumannii complex was the predominant species in our study (83.3\%). This $\mathrm{w}$ as similar in other studies like Nariz et al (98\%), De AS et al (84.6\%), Vinodkumar et al $(91.2 \%)$. This percentage was however lower in other studies like Arora et al $(56.52 \%)$, Mondal et al $(60 \%){ }^{8}$

In this study, Acinetobacter sepsis was found more in low birth weight babies (75\%) and preterm babies (58.3\%), which was similar to other studies. Nazir et al low birth weight $(81.6 \%)$, De AS et al (65.3\%). Preterm babies with sepsis in other studies were Nazir et al (77.5\%), De AS et al (69.1)..$^{10-12}$ It was also matching with other studies. ${ }^{8,9,11}$ Preterm infants have upto 3-5 fold higher risk of infection that full term infants as they are on prolonged intravenous drugs, ventilator support, or other invasive procedure that provides an opportunity for Acinetobacter species to gain entry. In this study increase in Acinetobacter septicemia was noted with prolonged ICU stay $(66.6 \%)$. Other studies did not show such high relation between prolonged stay and increased Acinetobacter infection, Nazir et al (45\%), De AS et al $(38.4 \%)$, Based on this findings it is important that we strengthen our infection control practices.
All Acinetobacter baumannii 7(100\%) strains were resistant to Carbapenems, similar pattern was noticed from other studies Nazir et al. MDR Acinetobacter is reported globally and causes most difficult HAIs to treat. MDR Acinetobacter species percentage in our study was $11(91.6 \%)$, Nazir et al also showed similar results (95.9\%), other studies showed low MDR percentage among Acinetobacter species De AS et al (53.75\%). ${ }^{12}$

\section{Conclusion}

Neonatal septicemia especially with MDR Acinetobacter is on the rise and is associated with increased morbidity and mortality. Continuous surveillance of isolates from neonatal septicemia, adherence to infection control policies and rational antibiotic usage will reduce the incidence of such infections. Since all babies had clinical features suggestive of septicaemia, the organism was considered to be significant. Acinetobacter spp is an important pathogen of nosocomial septicaemia in neonates. Source of infection for outbreaks of Acinetobacter septicemia have been traced to medical equipment, emphasizing the need for special attention to disinfection of shared items and etra care with respiratory care and wound care. Rational antibiotic use along with implementation of infection control policies are required for control of such infections.

\section{Source of Funding}

None.

\section{Conflict of Interest}

None.

\section{References}

1. Mahon CR, Lehman DC, Manuselis G. George Manuselis. Textbook of diagnostic microbiology ; 2007,. p. 489-491.

2. Bergogne-Berezin E, Towner KJ. Acinetobacter species as nosocomial pathogens: Microbiological, clinical, and epidemiological features. Clin Microbiol Rev. 1996;9:148-65. 
3. O A, A O. Prevalence and in-vitro antimicrobial susceptibility patterns of acinetobacter strains isolated from patients in intensive care units. J Int Med Res. 2003;31:272-80.

4. Khatua SP, Das AK, Chatterjee BD, Khatua S, Ghose B, et al. Neonatal septicaemia. Indian J Pediatr. 1986;53:509-14.

5. Gerner-Smidt P, Tjernberg I, Ursing J. Reliability of phenotypic tests foridentification of Acinetobacter species. J Clin Microbiol. 1991;29:277-82.

6. Clinical and Laboratory Standards Institute. ; 2012,.

7. Touati A, Achour W, Cherif A, Hmida HB, Afif FB, et al. Outbreak of Acinetobacter baumannii in a neonatal intensive care unit: Antimicrobial susceptibility and genotyping analysis. Ann Epidemiol. 2009; 19:372-380.

8. Arora U, Jaitwani J. Acinetobacter spp.: An emerging pathogen in neonatal septicaemia in Amritsar. Indian J Med Microbiol. 2006;24:81-81

9. Vinodkumar CS, Neelagund YF. Acinetobacter septicaemia in neonates. Indian J Med Microbiol. 2004;22:71-71.

10. A N. Multidrug-resistant Acinetobacter septicemia in neonates: A study from a teaching hospital of Northern India. J Lab Physicians. 2019;11:23-31.
11. Mondal GP, Raghvan M, Vishnu B, Srinivasan S. Neonatal septicemia among inborn and outborn babies in a referal hospital. Indian $J$ Pediatr. 1991;58:529-562.

12. Shete VB, Ghadage DP, Bhore AV. Acinetobacter Septicemia in Neonates Admitted to Intensive Care Units. J Lab Physicians. 2009;1(2):73-76.

\section{Author biography}

Ruturaj M Kolhapure Assistant Professor

\section{A. Ravi Shankar Reddy Professor}

Raj Kumar H.R.V Professor and HOD

Cite this article: Kolhapure RM, Reddy ARS, Kumar H.R.V R. Septicemia in neonates admitted to NICU with special reference to Acinetobacter species. Indian J Microbiol Res 2019;6(3):241-244. 